\title{
LA COMPETENCIA MEDIÁTICA Y LOS CURSOS MOOC EN ESTUDIANTES DE LA UTN:
} IMPLICACIONES, RETOS Y POTENCIALIDADES

\section{MEDIA COMPETITION AND MOOC COURSES IN STUDENTS OF THE UTN: IMPLICATIONS, CHALLENGES AND POTENTIALITIES}

Vivian Ojeda La Serna, MSc.

Máster en Estudio Sociales de la Ciencia y la Tecnología (Cuba). Docente investigadora de la Universidad Técnica del Norte, Ecuador. vojeda@utn.edu.ec

Andrea Verenice Basantes Andrade, MSc. Máster en Tecnología para la Gestión y Práctica Docente (Ecuador). Docente investigadora de la Universidad Técnica del Norte, Ecuador. avbasantes@utn.edu.ec

Mari Carmen Caldeiro Pedreira, PhD. Doctora en Comunicación y Educación (España). Docente investigadora de la Universidad Santiago de Compostela, España. mcarmen.caldeiro@usc.es

\section{ARTÍCULO DE INVESTIGACIÓN}

Recibido: 1 de septiembre de 2018.

Aceptado: 9 de noviembre de 2018.

\section{RESUMEN}

El desarrollo de la competencia mediática en la formación de los jóvenes universitarios permite abordar la recepción del contenido, los modos de interacción, el manejo de la tecnología y la producción del mensaje, desde una postura adecuada, responsable, crítica y autónoma. A través de la habilidad mediática podrán alcanzar las destrezas necesarias para aprovechar la gran oferta existente de "Cursos Masivos Abiertos" (MOOC) disminuyendo la tendencia de alta deserción de los mismos. En la Universidad Técnica del Norte de Ecuador (UTN), se llevó a cabo una investigación que busca analizar en estudiantes que finalizaron y no el curso la relación entre algunas dimensiones de la competencia mediática y el aprendizaje a través de 
MOOC. Mediante un enfoque mixto se hace hincapié en la competencia mediática, que les permite interactuar y comunicarse de manera autónoma tomando como base de estudio su experiencia en MOOC. Los datos indican que, en el contexto latinoamericano y ecuatoriano, en particular, aún queda mucho por hacer en cuanto a formación de jóvenes universitarios en las competencias que demanda la educación en la era digital.

Palabras clave: competencias mediáticas, competencia digital, MOOC, educación, alumnado universitario.

\section{ABSTRACT}

The development of media competence in the university students training allows us to refer to the reception of content. It also allow to be conscience of the interaction ways, the management of technology and the production of the message, from an adequate, responsible, critical and autonomous position. Students will be able to reach the necessary skills to take advantage of the great existing offer of "Open Massive Courses" (MOOC) through the media skill. This way of work looks for decrease the desertion of the same ones. In the Technical University of the North of Ecuador (UTN), an investigation was carried out in order to seeks the analyses in students who finished and students who don't finished the course. It looks for the relationship between some dimensions of media competence and learning through MOOC. It was used a mixed approach, emphasis it is focused on media competence, which allows them to interact and communicate autonomously based on their MOOC experience. The data indicate that in the Latin American context and Ecuadorian in particular, there is still much to be done in terms of training young university students in the competencies demanded by education in the digital age.

Keywords: media competence, Digital competence, MOOC, education, university students.

\section{INTRODUCCIÓN}

La omnipresencia mediática y la exponencial proliferación de pantallas y dispositivos tecnológicos nos adscriben, de forma irremediable a cambios metodológicos en el ámbito académico. Nuevas formas de enseñar que buscan conciliar el crecimiento formativo con el desarrollo laboral al que se suman diferentes necesidades fruto de la sociedad del siglo XXI. De forma especial a lo largo de la última década y de manera creciente durante el último lustro asistimos a un incremento casi generalizado del número de conexiones a la red, Internet se ha convertido en un gigante que provee de conexión y permite la interconexión. Tanto los nativos como los inmigrantes digitales necesitan conocer y dominar nuevas formas de 
comunicación que les convierten en «natitantes» (Fernández-García, Blasco-Duatis y Caldeiro-Pedreira, 2016). Para ello es necesario que se formen de manera continua y constante, una exigencia que se ha identificado a lo largo de los últimos años con el «life long learning» (Longworth, 2005) que favorece el alcance de la competencia crítica (Caldeiro y Aguaded, 2015), una habilidad que ha de desarrollar de forma general la audiencia, es decir, los hombres y mujeres de cualquier edad que conviven en la «sociedad multipantalla» (Pérez Tornero, 2007). El alcance de esta competencia con base en las dimensiones de la interacción y producción de contenidos de forma ética, permite la proliferación de «prosumidores» (Sánchez Carrero y Contreras Pulido, 2012), es decir, sujetos capaces de, no solo recibir, sino de comprender la información y los contenidos audiovisuales que reciben.

Esta realidad justifica e implica un cambio pedagógico inminente que flexibilice los tiempos de formación en función de la condición de los usuarios. Shaikh y Khoja (2012) afirman que los nuevos roles didácticos implican motivar al alumnado a tomar la propiedad y el control de su aprendizaje para alcanzar sus metas con éxito. Para ello surgen los MOOC «cursos masivos abiertos on-line» que permiten que el alumnado realice su inducción según su disponibilidad y que pueda conjugar la tarea formativa con la profesional. Se trata, siguiendo a Bartolomé Pina y Stieffens Kölh, (2015, p. 91) de, «una nueva forma de entornos virtuales de aprendizaje potenciados por la tecnología». Es un proceso social mediante el cual el ser humano aprender e internalizar conocimientos, valores, destrezas y habilidades a fin de cualificarse más y mejor.

\section{REVISIÓN TEÓRICA}

\subsection{El aprendizaje 3.0: más allá de lo convencional}

La adquisición de conocimientos trasciende fronteras, en este sentido el proceso de enseñanza-aprendizaje se concibe como un modo horizontal de construir el conocimiento en el cual el profesor es un guía que se comunica bidireccionalmente con su alumnado. Para el cumplimento de tal cometido se requiere de estrategias tecno pedagógicas que permitan gestionar, administrar, compartir, monitorear, evaluar y retroalimentar las diferentes actividades de formación (Basantes, Naranjo y Ojeda, 2018) cuya filosofía adecúe el mundo educativo a los cambios acaecidos en la sociedad (Gil y Martínez, 2018, p. 55), trascendiendo a las memorísticas centradas en el ámbito cognitivo por excelencia.

En este sentido, en la sociedad del conocimiento y la interactividad resulta impensable circunscribir la formación a un momento concreto y cerrado, precisa un saber, uso y dominio 
adecuado para gestionar debidamente las habilidades propias de la era digital (Cózar Gutiérrez, De Moya Martínez, Hernández Bravo y Hernández Bravo, 2016). Partiendo del Informe DeSeCo (OCDE, 2001) que plantea la enseñanza por competencias y teniendo en cuenta que, no solo aprendemos a aprender, sino que aprendemos a hacer y a estar, es necesario optar por nuevos modos de construcción del conocimiento que subrayen y afiancen el valor de la creatividad, el trabajo colaborativo y el alcance de la autonomía. Objetivos que resultan fácilmente alcanzables teniendo en cuenta que la educación virtual, a través de los cursos MOOC tiene como pilar básico no solo el desarrollo de las competencias básicas sino además el compartir experiencias en comunidad sin límites de espacio y tiempo.

En este sentido los MOOC se han convertido entre 2011-2013 en Europa en, formas de innovar y co-crear conocimientos (Ramírez Montoya y García Peñalvo, 2018), actividades de gran importancia que han revolucionado, de forma excepcional la educación superior. Por su parte, en continentes como el Latinoamericano ha sido necesario un poco más de tiempo para poder constatar datos.

\subsubsection{El desarrollo de habilidades mediáticas a través de los MOOC.}

La realidad certifica el cambio y el abandono de ciertos tabúes por parte de aquellas personas que consideran esta formación como elitista y como una formación que puede ocasionar brechas poblacionales. Más allá de ello, la realidad demuestra un ligero incremento de este tipo de cursos en diversos puntos geográficos. Este hecho, que inicialmente podía estar ligado a condiciones socio políticas concretas comienza a institucionalizarse. Una institucionalización que no se circunscribe únicamente al ámbito de la demanda sino también al de la oferta, esto es, hace años en algunos países resultaba poco común la existencia de MOOC y la gente que procedía de estos lugares únicamente estaba en contacto con estas formas de enseñanza como espectadores, es decir, como alumnado. Sin embargo, conforme avanza el tiempo la realidad cambia y comienza a ofertarse este tipo de formación en puntos del planeta más dispares. A lo largo de 2016, concretamente entre febrero y octubre se han producido en Latinoamérica en torno a 140 MOOC (Pérez, Maldonado y Morales, 2016), con lo cual se avisora un aporte importante en cuanto a esta modalidad dentro de los procesos de enseñanza-aprendizaje.

No obstante, los MOOC constituyen una oferta que continúan monopolizando plataformas con origen en Europa y EEUU tales como Red Educa, Miríada X, Ecolearning, Coursera, EdX o Udacity, entre otras. Espacios a través de los cuales es posible aprender, en lengua española y en ocasiones como Udacity, en inglés. 
El fenómeno MOOC acarrea una ingente cantidad de dudas, interrogantes que trascienden la forma y se centran en el fondo. Con frecuencia existen estudios de mercado que cuestionan el valor de este tipo de formación y el porqué del abandono de estos cursos. Frente a estos interrogantes que ponen en duda la calidad e incluso la valía de estos cursos conviene señalar algunas potencialidades.

En primer lugar, la formación online no se equipara a una carrera universitaria, sino que se concibe como un complemento que refuerza y especializa aspectos concretos. Además de esto, los cursos MOOC favorecen el contacto y el trabajo colaborativo de los usuarios quienes, en ocasiones y si surgen dudas, a través del foro se ayudan para poder avanzar y alcanzar el final del proceso.

Estudios anteriores refuerzan que los estudiantes para cursar MOOC tienen en cuenta cómo estos le aportan a su currículum, por lo que le dan valor a la institución que ofrece el curso y el costo del certificado. Por otra parte, el tiempo es una variable imprescindible en la evaluación o toma de decisiones sobre los MOOC a cursar, por lo que la duración del curso se convierte en un factor clave de decisión. El tema de la estética también es relevante, pues en la era digital hay mucha información multimedia, hipertextual, multimodal, etc., que pone al usuario, en este caso al estudiante, siempre en expectativa de la presentación de la información.

Asimismo, resulta innegable que el porcentaje de matriculados se reduce de forma notable al finalizar el curso. El motivo de este descenso no puede circunscribirse a un solo factor, sino que se debe a diversos factores como interés de los matriculados, el tiempo que pueden dedicarle o simplemente los contenidos que el curso, a priori, parece ofertar.

Por tanto y dado que en esta comunicación no es menester alcanzar una respuesta a tal interrogante, centraremos la atención en la valía de este tipo de formación puesto que, el mero hecho de matricularse en un curso ya supone un conocimiento y aplicación de la dimensión de la tecnología. Además de ello, indica que el alumnado es capaz de comunicarse, al menos de forma inicial y básica, a través de la tecnología y no solo esto, sino que encuentra en esta un valor educativo añadido.

Como parte de la interacción de los estudiantes con el conocimiento a través de las pantallas, existen una serie de aspectos que Ferrer y Piscitelli (2012) lo determinan como dimensiones e indicadores de las competencias mediáticas y que en este trabajo se consideran elementos 
importantes para tener un proceso exitoso cursando los MOOC. El interés de esta investigación se centra en algunos de ellos que se exponen a continuación, interrelacionándolos con los atributos estudiados.

Tabla 1. Dimensiones, indicadores de Competencia Mediática relacionados con los atributos de esta investigación.

\begin{tabular}{|c|c|c|c|}
\hline \multicolumn{2}{|c|}{ Dimensión } & \multirow[b]{2}{*}{$\begin{array}{l}\text { Indicador } \\
\text { Analizar y valorar mensajes } \\
\text { desde la perspectiva del } \\
\text { significado, sentido, } \\
\text { estructuras narrativas, } \\
\text { géneros y formatos. }\end{array}$} & \multirow{2}{*}{$\begin{array}{l}\text { Atributo } \\
\text { Criterio de selección del tema del } \\
\text { MOOC }\end{array}$} \\
\hline Lenguaje & Análisis & & \\
\hline & Expresión & $\begin{array}{l}\text { Elegir distintos sistemas de } \\
\text { representación y estilos en } \\
\text { función del contenido a } \\
\text { transmitir e interlocutor. }\end{array}$ & Participación en los foros \\
\hline \multirow[t]{2}{*}{ Tecnología } & Análisis & $\begin{array}{l}\text { Interaccionar de manera } \\
\text { significativa con medios que } \\
\text { permiten expandir las } \\
\text { capacidades mentales. }\end{array}$ & Materiales/Recursos \\
\hline & Expresión & $\begin{array}{l}\text { Manejar con corrección } \\
\text { herramientas comunicativas } \\
\text { en un entorno multimedial y } \\
\text { multimodal }\end{array}$ & Nivel tecnológico requerido \\
\hline \multirow[t]{2}{*}{$\begin{array}{l}\text { Procesos } \\
\text { de } \\
\text { recepción y } \\
\text { de } \\
\text { interacción }\end{array}$} & Análisis & $\begin{array}{l}\text { Seleccionar, revisar y } \\
\text { autoevaluar la propia dieta } \\
\text { mediática, en función de } \\
\text { criterios conscientes y } \\
\text { razonables. }\end{array}$ & $\begin{array}{l}\text { Hábitos de estudio, Gestión del } \\
\text { tiempo para estudiar mediante } \\
\text { MOOC }\end{array}$ \\
\hline & Expresión & $\begin{array}{l}\text { Interaccionar con personas } \\
\text { y con colectivos diversos en } \\
\text { entornos plurales y } \\
\text { multiculturales. }\end{array}$ & Motivación y seguimiento \\
\hline
\end{tabular}




\begin{tabular}{|c|c|c|c|}
\hline \multirow[t]{2}{*}{$\begin{array}{l}\text { Ideología y } \\
\text { valores }\end{array}$} & Análisis & $\begin{array}{l}\text { Conocer las fuentes de } \\
\text { información, evaluando su } \\
\text { fiabilidad y extrayendo } \\
\text { conclusiones críticas de lo } \\
\text { que se dice y se omite. }\end{array}$ & $\begin{array}{l}\text { Incidencia del profesor de la } \\
\text { asignatura que orientó el MOOC } \\
\text { como curso de apoyo }\end{array}$ \\
\hline & Expresión & $\begin{array}{l}\text { Usar nuevas herramientas } \\
\text { comunicativas de acuerdo } \\
\text { con valores cívicos y } \\
\text { democráticos, y bien del } \\
\text { medio social y natural. }\end{array}$ & Autonomía en el aprendizaje \\
\hline \multirow[t]{2}{*}{ Estética } & Análisis & $\begin{array}{l}\text { Cultivar el gusto por los } \\
\text { aspectos formales, no sólo } \\
\text { por lo que se comunica sino } \\
\text { también por la manera como } \\
\text { se comunica. }\end{array}$ & Materiales/Recursos \\
\hline & Expresión & $\begin{array}{l}\text { Producir mensajes } \\
\text { comprensibles que } \\
\text { incrementen personal o } \\
\text { colectivamente la } \\
\text { creatividad, originalidad y } \\
\text { sensibilidad. }\end{array}$ & Aportes del curso para su formación \\
\hline
\end{tabular}

Fuente: Adaptado de Ferrés y Piscitelli, 2015.

Entre las exigencias de la educación en esta era del conocimiento digital, se precisa de un estudiante comprometido y alejado de una actitud pasiva. En este estudio se ha intentado interrelacionar las dimensiones e indicadores que los académicos han definido para las competencias mediáticas, con atributos de los estudiantes para cursar con éxito el MOOC, como son el interés por elegir un tema, los hábitos de estudio que son necesarios porque reflejan el compromiso del estudiante con su formación académica, la autonomía que permite una correcta gestión del tiempo, una aceptación de la autoevaluación y los recursos que sugieren la adaptabilidad de nuevas formas de educación en el contexto digital en que hoy se convive.

Todos los elementos anteriores inciden en la experiencia académica de los estudiantes mediante MOOC. De forma general se puede afirmar que el mero conocimiento de estos cursos y el interés a la hora de matricularse forman un tándem que dibuja el perfil de la 
ciudadanía digital del siglo XXI. En la sociedad contemporánea se han de dominar formas de comunicación e interacción del entorno digital, y sobre todo desarrollar la autonomía y actitud crítica, (Caldeiro, 2014 y Aguaded y Caldeiro, 2013).

\section{MATERIALES Y MÉTODOS}

\subsection{Metodología}

Esta investigación de fundamenta en el enfoque mixto (cuali-cuantitativo) de corte explicativo, documental y de campo. El objetivo de la investigación es analizar en estudiantes que finalizaron y no finalizaron los MOOC (orientados como apoyo de asignaturas de la modalidad presencial), aspectos que favorecieron estos resultados teniendo como base algunas dimensiones de la competencia mediática. Para el efecto se establecieron tres fases: recolección de la información, muestra y diseño de la investigación, finalmente desarrolloprocedimiento.

\subsubsection{Fase 1 - Recolección de la información}

La investigación documental implementada a través del uso de técnicas bibliográficas posibilitó cubrir la primera fase de la investigación tanto para el ordenamiento de la información como para la construcción del marco teórico con carácter crítico-interpretativo (Vargas, Higuita y Muñoz, 2015). La recopilación de la información se llevó a cabo en las diferentes bases de datos indexadas a la biblioteca virtual de la Universidad Técnica del Norte tales como Scopus, SCimago, Scielo, Ebsco, ProQuest entre otras. Se obtuvieron 43 referencias relacionadas con estudios que contienen las palabras claves MOOC, competencias mediáticas, e-learning, aprendizaje; a fin de visibilizar la relación entre competencia mediática y aprendizaje a través de MOOC.

\subsubsection{Fase 2 - Muestra y diseño de la investigación}

El universo o población de estudio estuvo conformada por el censo de los estudiantes de los primeros y segundos semestres de las carreras de Psicología Educativa, Educación Inicial y Educación Básica, lo cual suman 168 estudiantes a los que se les orientó matricularse y cursar un MOOC como complemento del aprendizaje que están adquiriendo. Se construyó la matriz de relación (objetivos, variables, indicadores y técnicas) a fin de diseñar el instrumento de investigación que permita recoger los datos más significativos para establecer la relación del 
objeto de estudio. El cuestionario piloto estuvo conformado por 17 preguntas de selección múltiple (tipo cerradas), este fue validado por 4 expertos de la Universidad Técnica del Norte en el área de e-learning, quienes evaluaron la pertinencia, estructura, redacción y claridad de cada una de las preguntas; el instrumento final se conformó de 11 preguntas; sin embargo, para determinar la confiabilidad y validez del instrumento de se realizó una prueba piloto con 21 estudiantes, 7 de cada carrera tomados al azar, quienes contestaron la encuesta. Según Herrera (1998) la escala que se detalla en la Tabla 2 permite determinar la confiabilidad del instrumento.

Tabla 2: Escalas de confiabilidad.

\begin{tabular}{ccc}
\hline Límite inferior & Límite superior & Categorías \\
\hline Menor a 0,53 & 0,53 & Confiabilidad Nula \\
0,54 & 0,59 & Confiabilidad Baja \\
0,60 & 0,65 & Confiable \\
0,72 & 0,71 & Muy Confiable \\
1,0 & 0,99 & $\begin{array}{c}\text { Excelente } \\
\text { Confiabilidad }\end{array}$ \\
\end{tabular}

Fuente: Elaboración propia.

Con un valor de 0.8226 y de acuerdo con la escala aplicada se puede afirmar que el instrumento en lo que corresponde a las variables cualitativas nominales tiene una Excelente confiabilidad y puede ser aplicado.

Los datos obtenidos con el instrumento de investigación (cuestionario) fueron procesados en una hoja de cálculo de Microsoft Excel 2016 y posteriormente se utilizó el Paquete Estadístico de Investigación Social (SPSS) donde a través del cruce de variables se logró establecer el comportamiento de los estudiantes en el ámbito digital, sin provocar cambios en su conducta, lo cual se proyecta para futuras investigaciones los resultados de la investigación.

También se realizaron focus group en la totalidad de los 6 paralelos estudiados, para lo cual se dividieron los estudiantes en varios grupos de hasta 12 alumnos. A partir de una guía de preguntas se abordó, con los grupos, el debate sobre su experiencia en los MOOC, 
principalmente dirigido a cuáles fueron los aspectos negativos y positivos que consideran tras su experiencia.

\subsubsection{Fase 3 - Desarrollo-procedimiento}

La investigación tuvo dos momentos, durante los cuales transcurrieron los 4 pasos que conformaron el desarrollo-procedimiento.

Un primer momento fue al inicio del semestre donde se dio una introducción sobre MOOC y su aporte a la formación profesional desde la modalidad presencial que están recibiendo esos grupos. Consecutivamente se les dio orientaciones para que refuercen sus conocimientos mediante la matrícula y aprobación de un curso MOOC, se les mostró varias plataformas existentes para que a libre criterio eligieran la plataforma y el MOOC. En un segundo paso los propios estudiantes expresaron sus criterios de selección del MOOC.

Antes de terminar el semestre tuvo lugar el segundo momento de la investigación, se aplicó la encuesta online expuesta en la Fase 2 (Muestra y diseño de la investigación), y se llevaron a cabo los focus group, con la finalidad de recolectar la información necesaria para llevar a cabo el análisis de su experiencia en los MOOC y su puesta en práctica o no de competencias mediáticas para comunicarse, interactuar y producir información.

Un tercer momento estuvo dado por el análisis de la información, para lo cual se utilizó el software SPSS para el análisis de los datos recogidos en la encuesta, y la búsqueda de correlación entre las variables estudiadas. Un cuarto momento sirvió para transferir los resultados de la investigación a los estudiantes involucrados.

\section{ANÁLISIS DE RESULTADOS}

Los resultados que más sobresalen en esta investigación son los identificados con la inscripción y participación masiva de los estudiantes, un dato que ha resultado alentador a pesar de los altos índices de deserción en esta modalidad de estudios. Los estudiantes la hora de seleccionar un MOOC para fortalecer, actualizar y perfeccionar sus conocimientos tienen en cuenta algunos criterios que les permiten decidir por cuál opción optar, en primera instancia revisan el tema (41,9\%), seguido por la duración del curso (20.6\%) tiempo, número de horas; 
luego consideran la universidad que oferta la capacitación (prestigio institucional) $(17,7 \%)$ y finalmente el costo de la certificación (9,7\%).

El 90,5\% de los estudiantes han seguido cursos mediante MOOC, de este segmento el 69,8\% ha tomado el curso mediante la plataforma de Miriadax, seguido por Coursera (22,2\%), Red Educa (3\%) y otras plataformas (5\%). Los temas con mayor preferencia son los que se relacionan con la carrera que actualmente se están formando (76,3\%), mientras que el 23,7\% toman los cursos por interés personal que no es a fin a su carrera profesional.

Además de ello, la experiencia académica de los estudiantes mediante MOOC ha sido satisfactoria $(71,5 \%)$, el $27 \%$ poco satisfactoria y el $1.5 \%$ nada satisfactoria.Asimismo, el tiempo que le dedicaron para estudiar bajo esta modalidad tiene mayor incidencia en la revisión semanal $(40,3 \%)$, seguido de las personas que lo hacen de forma quincenal (29\%), cabe señalar que el $25,8 \%$ de los estudiantes trabajan las diferentes actividades propuestas en el curso de forma mensual, y muy difícilmente revisan los contenidos diariamente $(4,8 \%)$. Así mismo, se determinó que el horario de acceso con mayor concurrencia a la plataforma MOOC es por la noche $79.7 \%$, el acceso por la tarde tiene una afluencia inferior $18,6 \%$ y más aún en la mañana $1,7 \%$.

En relación con la valoración de los materiales y recursos que se usan y su proximidad con la competencia digital, en el desarrollo del aprendizaje mediante MOOC se ha comprobado que, los que finalizaron el curso encuentran muy satisfactorio el uso de videos (29\%), seguido por la participación e interacción en los foros (25\%). A diferencia de los que no finalizaron el curso, manifiestan que son muy satisfactorios los test autoevaluativos seguido por las Lecturas de los contenidos adicionales o descargas. Cabe señalar que el menos valorado corresponde a los ejercicios y actividades. Ver Tabla 1.

Tabla 2. Valoración de materiales y recursos de los MOOC.

\begin{tabular}{|c|c|c|c|c|c|c|c|c|c|c|}
\hline \multirow{2}{*}{ Materiales-Recursos } & \multicolumn{2}{|c|}{$\begin{array}{c}\text { Muy } \\
\text { Satisfactori } \\
0\end{array}$} & \multicolumn{2}{|c|}{$\begin{array}{c}\text { Satisfact } \\
\text { orio }\end{array}$} & \multicolumn{2}{|c|}{$\begin{array}{c}\text { Medianamente } \\
\text { Satisfactorio }\end{array}$} & \multicolumn{2}{|c|}{$\begin{array}{c}\text { Poco } \\
\text { Satisfactori } \\
0\end{array}$} & \multicolumn{2}{|c|}{$\begin{array}{c}\text { Nada } \\
\text { Satisfactori } \\
0\end{array}$} \\
\hline & 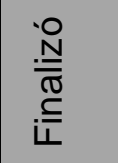 & 운 & 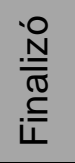 & 운 & 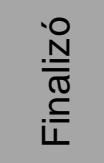 & 은 $\stackrel{\stackrel{N}{N}}{\stackrel{N}{=}}$ & 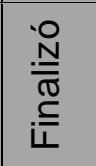 & 올ำ & $\begin{array}{l}\stackrel{N}{N} \\
\stackrel{N}{\widetilde{N}}= \\
i=\end{array}$ & 온 \\
\hline Videos Didácticos & $29 \%$ & $0 \%$ & $\begin{array}{l}41 \\
\%\end{array}$ & $\begin{array}{l}13 \\
\%\end{array}$ & $8 \%$ & $8 \%$ & $2 \%$ & $0 \%$ & $0 \%$ & $0 \%$ \\
\hline
\end{tabular}




\begin{tabular}{|c|c|c|c|c|c|c|c|c|c|c|}
\hline $\begin{array}{l}\text { Ejercicios/ } \\
\text { Actividades }\end{array}$ & $17 \%$ & $0 \%$ & $\begin{array}{l}46 \\
\%\end{array}$ & $\begin{array}{l}19 \\
\%\end{array}$ & $10 \%$ & $6 \%$ & $0 \%$ & $0 \%$ & $2 \%$ & $0 \%$ \\
\hline Test autoevaluativo & $21 \%$ & $2 \%$ & $\begin{array}{l}29 \\
\%\end{array}$ & $\begin{array}{l}21 \\
\%\end{array}$ & $8 \%$ & $16 \%$ & $2 \%$ & $2 \%$ & $0 \%$ & $2 \%$ \\
\hline Participación en los foros & $25 \%$ & $0 \%$ & $\begin{array}{l}44 \\
\%\end{array}$ & $\begin{array}{l}11 \\
\%\end{array}$ & $5 \%$ & $10 \%$ & $2 \%$ & $2 \%$ & $0 \%$ & $2 \%$ \\
\hline $\begin{array}{l}\text { Lectura de los contenidos } \\
\text { adicionales o } \\
\text { descargables }\end{array}$ & $24 \%$ & $2 \%$ & $\begin{array}{l}37 \\
\%\end{array}$ & $\begin{array}{l}13 \\
\%\end{array}$ & $8 \%$ & $14 \%$ & $0 \%$ & $2 \%$ & $2 \%$ & $0 \%$ \\
\hline
\end{tabular}

Fuente: Elaboración propia

A fin de determinar la fidelización de este sistema de aprendizaje (MOOC) se indagó a los que si terminaron el curso si recomendarían su uso para la actualización y formación personal, el $85,2 \%$ de los estudiantes expresaron que si lo harían, y como tienen dominio de la competencia digital tienen una valoración más positiva para optar por esta modalidad $(90 \%)$ nuevamente, les resulta atractivo el uso de las TIC en las clases de la universidad. El $14.8 \%$ no recomendarían el MOOC porque su avance académico no fue suficiente para establecer una valoración adecuada, no todo es positivo en la aplicación de los MOOC, aún falta mucho por hacer en cuanto a preparar competencias en los estudiantes universitarios para que sean capaces de asumir estas nuevas formas de aprendizaje y puedan completar el curso deseado.

Sin embargo, del segmento que no finalizó el curso en el MOOC el 86,4\% volvería a tomar un curso bajo esta modalidad. En este mismo estrato los factores que incidieron en los estudiantes para terminar el curso, el de mayor relevancia es la incidencia del profesor de la asignatura presencial $(59,80 \%)$, seguido de los hábitos de estudios (27\%) y gestión del tiempo $(22,0 \%)$ y en su orden en un nivel de significancia menor por pérdida de interés, el contenido no aporta a su formación, limitado nivel tecnológico requerido y finalmente la motivación y seguimiento del estudiante por parte del tutor del MOOC.

La comparativa entre los estudiantes que finalizaron y no el curso, confirma que la gestión del tiempo es un factor que incide notablemente en la organización y cumplimiento de las diferentes actividades del MOOC, el de menor relevancia es el nivel tecnológico requerido. De igual modo, se busca conocer cuál ha sido el aporte del curso para la formación del alumnado que lo ha finalizado; en este caso según señalan los datos, el interés por obtener la certificación oficial del curso depende de la universidad que lo acredite. en un $58,1 \%$ de los casos mientras que el interés en mejorar su currículum es el factor determinante para el 24,2\% y que se exija en el trabajo centra el interés del $17,7 \%$ del total de la muestra participante 
En este sentido, la información que arrojó la encuesta realizada, coincide con los resultados de los focus group realizados a los estudiantes de primer y segundo nivel de las carreras estudiadas.

Completa el análisis de datos, el cruce de variables que se ha realizado utilizando los resultados cuantitativos de la encuesta en el programa SPSS.

Tabla 3. Correlaciones

\begin{tabular}{|c|c|c|c|c|c|c|c|c|c|c|}
\hline & $\begin{array}{l}\text { Atribución de } \\
\text { Éxito a } \\
\text { Hábitos de } \\
\text { estudio } \\
\text { Gestión del } \\
\text { tiempo para } \\
\text { estudiar } \\
\text { mediante } \\
\text { MOOC }\end{array}$ & $\begin{array}{c} \\
\\
\text { Final } \\
\text { izaci } \\
\text { ón } \\
\text { del } \\
\text { MO } \\
\text { OC }\end{array}$ & $\begin{array}{c}\text { Atribuci } \\
\text { ón del } \\
\text { Éxito a } \\
\text { Incidenc } \\
\text { ia del } \\
\text { profesor } \\
\text { de la } \\
\text { asignat } \\
\text { ura que } \\
\text { orientó } \\
\text { el } \\
\text { MOOC } \\
\text { como } \\
\text { curso } \\
\text { de } \\
\text { apoyo }\end{array}$ & $\begin{array}{l}\text { Atribu } \\
\text { ción } \\
\text { de } \\
\text { Éxito } \\
\text { a } \\
\text { Partici } \\
\text { pación } \\
\text { en los } \\
\text { foros }\end{array}$ & $\begin{array}{c}\text { Atribu } \\
\text { ción } \\
\text { del } \\
\text { Éxito } \\
\text { a } \\
\text { Motiva } \\
\text { ción y } \\
\text { segui } \\
\text { mient } \\
\text { o }\end{array}$ & $\begin{array}{c}\text { Atribu } \\
\text { ción } \\
\text { del } \\
\text { Éxito } \\
\text { a } \\
\text { Nivel } \\
\text { tecnol } \\
\text { ógico } \\
\text { requer } \\
\text { ido }\end{array}$ & $\begin{array}{c}\text { Atribu } \\
\text { ción } \\
\text { de } \\
\text { Éxito } \\
\text { al } \\
\text { Materi } \\
\text { ales/R } \\
\text { ecurs } \\
\text { os }\end{array}$ & $\begin{array}{c}\text { Atribu } \\
\text { ción } \\
\text { de } \\
\text { Éxito } \\
\text { a } \\
\text { Criteri } \\
\text { o de } \\
\text { selecc } \\
\text { ión del } \\
\text { tema } \\
\text { del } \\
\text { MOO } \\
\text { C }\end{array}$ & $\begin{array}{c}\text { Atribu } \\
\text { ción } \\
\text { de } \\
\text { Éxito } \\
\text { a } \\
\text { Auton } \\
\text { omía } \\
\text { en el } \\
\text { apren } \\
\text { dizaje }\end{array}$ & $\begin{array}{c}\text { Au } \\
\text { Atrib } \\
\text { ución } \\
\text { de } \\
\text { Éxito } \\
\text { a } \\
\text { Aport } \\
\text { es } \\
\text { del } \\
\text { curso } \\
\text { para } \\
\text { su } \\
\text { form } \\
\text { ación }\end{array}$ \\
\hline $\begin{array}{ll}\text { Finali } & \text { Correlació } \\
\text { zació } & \mathrm{n} \quad \mathrm{de} \\
\mathrm{n} \text { del } & \text { Pearson } \\
\text { MOO } & \text { Sig. } \\
\mathrm{C} & \text { (bilateral) } \\
& \mathrm{N}\end{array}$ & $\begin{array}{r}, 000 \\
428\end{array}$ & 428 & $\begin{array}{r}, 000 \\
428\end{array}$ & $\begin{array}{r}, 000 \\
428\end{array}$ &, $199^{* *}$ &, $186^{* *}$ & $\begin{array}{l}, 001 \\
428\end{array}$ &, $160^{* *}$ & ,316 &, $158^{* \star}$ \\
\hline
\end{tabular}

Fuente: Elaboración propia.

La correlación de la Finalización del MOOC con las demás variables es estadísticamente significativa al $(p<0,01)$, excepto con la Atribución del Éxito a Nivel tecnológico requerido, 
donde no es significativa la correlación $(p>0,05)$. La Finalización del MOOC tiene una correlación positiva y alta con Atribución del Éxito a Incidencia del profesor de la asignatura que orientó el MOOC como curso de apoyo $(r=, 576)$ y Atribución de Éxito a Hábitos de estudio, Gestión del tiempo para estudiar mediante MOOC ( $r=, 547)$. La Finalización del MOOC con respecto a Atribución de Éxito a Autonomía en el aprendizaje tiene relación positiva y media ( $r$ entre 0,3 y 0,5$)$. El resto de las variables tienen una relación positiva y baja con respecto a la finalización de los MOOC $(r<0,3)$. Los estudiantes que Finalizaron el MOOC son los que atribuyen su éxito fundamentalmente a la incidencia del profesor de la asignatura que orientó el MOOC, tienen hábitos de estudio y gestionan su tiempo para estudiar en esa modalidad de aprendizaje y en menor medida tienen autonomía.

\section{DISCUSIÓN DE RESULTADOS}

La integración de códigos del mundo digital al entorno educativo universitario podría, a priori, brindar algún tipo de garantía a los procesos de enseñanza-aprendizaje, sin embargo, la realidad se opone a esta hipótesis. En este sentido, una ingente cantidad de investigaciones se enfocan al papel del alumnado en los nuevos entornos educativos, siendo un punto común el desarrollo de la competencia digital demostrado en el uso de tecnologías y dispositivos para fortalecer las estrategias de aprendizaje.

En la investigación realizada en alumnos de las carreras de Psicología, Educación Inicial y Educación Básica de la Universidad Técnica del Norte, el entorno digital permite integrar a los procesos de enseñanza modos de comunicación inmediata y comunitaria (Caldeiro y Aguaded, 2015). En el caso de estos estudiantes universitarios se abordó la experiencia del uso de MOOC como refuerzo dentro de las asignaturas que cursan, por lo que se integran aspectos propios del ecosistema comunicativo y mediático que los estudiantes usan en su vida social, al entorno educativo.

Sin embargo, el acercamiento a estos cursos mayoritariamente fue por inducción docente más no por interés propio, con lo cual se evidencia el reto que supone la competencia digital para la comunicación fuera del aula, pues dentro de esta se tendrían que valorar otros factores. La búsqueda de estrategias de desarrollo de la autonomía en los estudiantes universitarios, debe ser un foco de interés necesario en esta era digital donde se vive y se estudia prácticamente dentro de las pantallas. En este estudio se demuestra que los universitarios del entorno estudiado, aún prefieren la presión ejercida por el docente lo cual se asemeja más a la pasada era conductista. 
En el estudio realizado, la mayoría de estudiantes terminó el curso de forma satisfactoria en contraposición a lo que varios autores han afirmado en cuanto al alto índice de deserción; lo cual se explica que la inducción y seguimiento de los docentes que realizaron esta propuesta influyó en los estudiantes para cumplir con el objetivo. En consonancia con Kizilcec, Piece y Schneider (2013) y Hollands y Tirthali (2014), para que un MOOC sea exitoso académicamente requiere de alumnos automotivados para el aprendizaje y con un alto nivel de compromiso hacia el mismo. Sin embargo, a partir de la autonomía de cada estudiante eligieron el tema del curso. La certificación queda en segundo plano cuando se aprende por iniciativa personal, la cualificación tiene mayor relevancia que la acreditación.

Por otro lado y en concordancia con los resultados obtenidos en la investigación realizada por Hernández Carranza, Romero Corella \& Ramírez Montoya (2015) los escenarios de aprendizaje abierto demandan mayores habilidades digitales para interactuar con los participantes, promover sinergias y alcanzar los objetivos educativos, de esta manera el alumnado que finalizó el MOOC demostró mayor dominio de competencia mediática, aún monitoreado por el profesor de la asignatura presencial, fue capaz de asimilar los contenidos, interactuar, mantenerse motivado y producir contenido para cumplir las tareas y la autoevaluación de forma autónoma. De forma especial a lo largo de los últimos años se cuenta con una gran oferta de MOOC en Internet, siendo las plataformas que presentan un mayor número de cursos seleccionados por los estudiantes Miriadax y Coursera. Estas plataformas dieron respuesta al interés de los alumnos, en un momento en el cual, según afirman Gil y Martínez (2018, p. 46), en las propuestas de MOOC "el alumnado elige el recorrido que se dispone a seguir según su propia disponibilidad e interés", los estudiantes seleccionaron los cursos principalmente siguiendo los criterios de selección del tema, tiempo de duración y la institución.

La disposición de tiempo es un aspecto al que se le debe tener en cuenta, pues los estudiantes no suelen dedicarle horas diarias al estudio en los MOOC, su disponibilidad se reduce a revisar los recursos semanal o quincenalmente. Las competencias de los estudiantes para asimilar las propuestas de aprendizaje a través de MOOC, sigue siendo una asignatura pendiente, teniendo en cuenta que después matriculados es muy visible la alta tasa de abandono de los cursos.

De la totalidad de estudiantes que culminaron los cursos buena parte de ellos consideran muy satisfactorio tener hábito de estudio y saber gestionar el tiempo para estudiar, además consideraron favorable el uso de los videos y la participación e interacción en foros. Esto dirige a las formas de comunicación e interacción en las redes sociales en las cuales los estudiantes 
invierten gran parte de su tiempo en la actualidad. En correspondencia, Pérez, Negre y Lizana (2017) a través de una investigación en la Universidad de Islas Baleares, parten de la idea de que la popularidad del uso de las redes sociales, presagian el éxito de comunidades virtuales. Estos autores evalúan y plantean que en ese ámbito los estudiantes gustan más de noticias académicas, más bien en el caso de compartir información y difundir actividades académicas; en segundo orden se interesan por las noticias culturales. Se recalca la importancia del paso de "la Universidad unidireccional hacia la Universidad multidireccional en la que el alumno asume un papel activo y la autorregulación del aprendizaje emerge como una exigencia en una sociedad con un marcado acento tecnológico" (Pérez, Negre y Lizana, 2017:512). Otro ejemplo que visualiza la predisposición de los estudiantes a la interactividad virtual en la enseñanza lo muestra Boza y Conde, quienes realizaron una investigación en la Universidad de Huelva (2017) resultando que los estudiantes tienen diferentes estilos de uso de la web 2.0, haciendo mejor uso los que estudian carreras de las ramas de ciencias sociales; (en la presente investigación también están involucradas carreras de ciencias sociales); no obstante, recalcan la necesidad de formar actitudes positivas hacia la web 2.0. Respecto a las competencias de los estudiantes para enfrentar esa interacción y el MOOC, en general implica que los mensajes educativos y la participación en foros deje de manejarse de manera acrítica y pasiva, y pasen a convertirse los estudiantes en prosumidores, quienes más allá de recibir el conocimiento, lo comprenden y producen. En concordancia Rodrigo Cano, Iglesias Onofrio, y Aguaded, (2017) hablan de "aprender a colaborar y de colaborar para aprender", en una investigación realizada en las universidades de Cádiz y de Sevilla.

También en la Universidad del País Vasco se realizó una investigación sobre la utilización de las redes sociales en los MOOC. Castaño, Maiz y Garay (2015) afirman que "todavía la mayoría de los potenciales participantes de MOOC no están totalmente preparados para el aprendizaje mediante la interacción mutua total y poco dirigida por el profesorado", lo cual coincide con los resultados de este estudio, aun así, las deducciones de su estudio corroboraron que el uso de las redes sociales como estrategia de participación colaborativa en un MOOC incide de manera positiva en la tasa de finalización de los estudiantes, así como en su motivación.

Un último aspecto a abordar es que la autoevaluación en los MOOC podría ser una fortaleza dentro de la autonomía que deben lograr los estudiantes, sin embargo, en esta investigación se dibuja como uno de sus motivos de deserción. Coincide con Martín (2015, 31), quien plantea que, "al no existir la figura de un tutor presencial, resulta difícil para gran parte del alumnado, puesto que no están acostumbrados a este tipo de autoevaluación". 


\section{CONCLUSIONES}

Las tecnologías de la información y las comunicaciones para la educación deben seguir desarrollándose y contribuyendo a una educación superior acorde a la era digital. Esta investigación defiende la postura mencionada ya por otros autores (Caldeiro, Yot y Castro, 2018, p.107) de la "urgente necesidad de una alfabetización mediática", en este caso aludiendo a la mostrada incapacidad de los estudiantes de culminar un MOOC si no es que tienen al profesor de la asignatura como elemento conductista, dejando ver una falta de autonomía, autogestión del tiempo y deficientes hábitos de estudio.

La literatura revisada y los datos extraídos de la experiencia realizada en la Universidad Técnica del Norte confirman que los MOOC se pueden incorporar como complementos de las asignaturas en la enseñanza universitaria. En correspondencia, la estrategia educativa de reforzar las asignaturas con MOOC fue considerada por el alumnado como satisfactoria y lo recomendarían a otros estudiantes, aunque hay que señalar que no ha sido seguida hasta el final por la totalidad del alumnado, aunque en este estudio este indicador resultó moderado dado a que un poco más de la mitad de los estudiantes si terminó el curso. Además de esto y como se ha demostrado en la investigación, tuvo gran peso el seguimiento que dio a los estudiantes de manera presencial el profesor de la asignatura que indicó los MOOC como apoyo para el aprendizaje.

En este sentido puede indicarse que la competencia mediática y los MOOC, más allá de sus potencialidades, plantean retos para el profesorado. Por todo ello, en el campo de la formación académica y el aprendizaje es necesario continuar con la incorporación de nuevas formas de hacer, con protagonismo tecnológico en el caso de los entornos virtuales de aprendizaje, pero a la par se deben perpetuar los valores y competencias necesarias para que los estudiantes sean ciudadanos responsables, críticos y autónomos.

\section{REFERENCIAS BIBLIOGRÁFICAS}

Bartolomé, A.R. \& Steffens, K. (2015). Are MOOCs Promising Learning Environments?. [¿Son los MOOC una alternativa de aprendizaje?]. Comunicar, 44, 91-99. https://doi.org/10.3916/C44-2015-10

Basantes, A. V., Naranjo, Miguel E., \& Ojeda, V. (2018). Metodología PACIE en la Educación Virtual: una experiencia en la Universidad Técnica del Norte. Formación universitaria, 11(2), 35-44. https://dx.doi.org/10.4067/S0718-50062018000200035 
Boza, A. y Conde, S. (2017). La Web 2.0 en la universidad de Huelva. Estilos de uso educativo. Revista Complutense de Educación, $28 \quad$ (3), 867-906. http://dx.doi.org/10.5209/RCED.2017.v28.n3.50501

Caldeiro, M.C., \& Aguaded, I. (2015). Alfabetización comunicativa y competencia mediática en la sociedad hipercomunicada. Revista Digital de Investigación en Docencia Universitaria, 9(1), 37-56. Recuperado de http://revistas.upc.edu.pe/index.php/docencia/article/view/379/387

Castaño Garrido, C.; Maiz Olazabalaga, I y Garay Ruiz, U. (2015). Redes sociales y aprendizaje cooperativo en un MOOC. Revista Complutense de Educación, 26 (1), 119-139. http://dx.doi.org/10.5209/rev RCED.2015.v26.46328

Cózar Gutiérrez, R., Moya Martínez, D., María, V., Hernández Bravo, J. A., \& Hernández Bravo, J. R. (2016). Conocimiento y Uso de las Tecnologías de la Información y las Comunicaciones (TIC) según el Estilo de Aprendizaje de los Futuros Maestros. Formación Universitaria, 9(6), 105-118. doi:10.4067/S071850062016000600010

Gil Quintana, J. \& Martínez Pérez, J. (2018). El empoderamiento del alumnado en los sMOOC. Revista Complutense de Educación, $29 \quad$ (1), 43-60. http://dx.doi.org/10.5209/RCED.51932

Fernández García, N., Blasco Duatis, M., \& Caldeiro Pedreira, M.C. (2016). Communication and Education by Transmedia. Report on ICT skills in four secondary schools in Europe. Cuadernos Artesanos de Comunicación.

Ferres, J. y Piscitelli, A. (2012). La competencia mediática: propuesta articulada de dimensiones e indicadores. Comunicar, 38, 75-82. DOI: dx.doi.org/10.3916/C38-201202-08

Ferrés, J., \& Piscitelli, A. (2015). La competencia mediática: Propuesta articulada de dimensiones e indicadores. Revista Luciérnaga-Comunicación, 4(7), 72-79. Recuperado de http://revistas.elpoli.edu.co/index.php/luc/article/view/286/254 
Hernández Carranza, E. E., Romero-Corella, S. I., \& Ramírez-Montoya, M. S. (2015). Evaluación de competencias digitales didácticas en cursos masivos abiertos: Contribución al movimiento latinoamericano. Comunicar, 22(44). DOI http://dx.doi.org/10.3916/C44-2015-09

Herrera, A. (1998). Notas sobre Psicometría. Bogotá: Universidad Nacional de Colombia.

Hollands, F., y Tirthali, D. (2014). Resource Requirements and Costs of Developing and Delivering MOOCs. The International Review of Research in Open and distance learning, 15(5), 113-133. Recuperado de http://www.irrodl.org/index.php/irrodl/article/view/1901/3069?

Kizilcec, R., Piece, C., y Schneider, E. (2013). Deconstructing disengagement: Analyzing learner subpopulations in massive open online courses. The 3rd Proceedings Of the Learning Analytics \& Knowledge Conference, Leuven, Belgium. Recuperado de http://www.stanford.edu/ cpiech/bio/papers/deconstructingDisengagement.pdf

Longworth, N. (2005). El aprendizaje a lo largo de la vida en la práctica. Barcelona: Paidós.

Martín, A. J. (2015). Opinión del alumnado acerca de los MOOC. Un estudio de caso. Recuperado

de https://riuma.uma.es/xmlui/bitstream/handle/10630/11214/TD Argote Martin.pdf?seq uence $=1$ \&isAllowed $=y$

OCDE (2001). Definition and selection of competencies (DeSeCo),: Theoretical and conceptual foundations. Strategy paper. Documento de internet disponible en www.deseco.admin.ch

Caldeiro Pedreira, MC., \& Aguaded, I. (2015). " Estoy aprendiendo, no me molestes" la competencia mediática como forma de expresión crítica de nativos e inmigrantes digitales. Redes. com: revista de estudios para el desarrollo social de la Comunicación, (12), 26-45. doi: 10.15213/redes.n12.p26

Caldeiro Pedreira, MC., Yot Domínguez, C. ., \& Castro Zubizarreta, A. C. (2018). Detección de buenas prácticas docentes de uso de dispositivos móviles en primaria a través del análisis documental. Prisma Social: revista de investigación social, (20), 58-75. 
Pérez Garcias, A.; Tur, G.; Negre Bennàssar, F. y Lizana, A. (2017). Factores de éxito de las comunidades virtuales universitarias basadas en redes sociales. Análisis de XarFED. Revista Complutense de Educación, $28 \quad$ (2), 497-515. http://dx.doi.org/10.5209/rev RCED.2017.v28.n2.49568

Pérez Tornero, J. M. (2007). Comunicação e educação na sociedade da informação. Novas linguagens e consciência crítica. Porto: Porto Editora.

Pérez Sanagustín, M., Maldonado, J. J., \& Morales, N. (2016). Estado del arte de adopción de MOOCs en la Educación Superior en América Latina y Europa. MOOC-Maker Constr. Manag. Capacit. MOOCs High. Educ, 1.

Ramírez Montoya, M.S. \& García Peñalvo, F. (2018). Co-creation and open innovation: Systematic literature review. [Co-creación e innovación abierta: Revisión sistemática de literatura]. Comunicar, 54, 09-18. https://doi.org/10.3916/C54-2018-01

Rodrigo Cano, D., Iglesias Onofrio, M., \& Aguaded, I. (2017). Metodologías participativas en la nube: la" g-Google" vs. la" Generación X" en la Web 2.0 1/Participatory methodologies in the cloud computing: the" g-Google" vs. the" X Generation" on the Web 2.0. Revista Complutense de Educación, 28(1), 223. http://dx.doi.org/10.5209/rev RCED.2017.v28.n1.49245

Sánchez, J. \& Contreras, P. (2012). De cara al prosumidor: producción y consumo empoderando a la ciudadanía 3.0. Icono 14, 10 (3); pp. 62-84. Recuperado de http://dx.doi.org/10.7195/ri14.v10i3.210

Shaikh , K. \& K Hoja , S. (2012). Role of Teacher in Personal Learning Environments. Digital Education Review, 21, 23-32. (http://goo.gl/oU3QOR) (19-03-2014).

Vargas, M. G., Higuita, C. G., \& Muñoz, D. A. J. (2015). El estado del arte: una metodología de investigación. Revista Colombiana de Ciencias Sociales, 6(2), 423-442. Recuperado de https://doi.org/10.21501/22161201.1469 